\title{
Determinantes do Investimento Estrangeiro Direto no Brasil: uma aplicação do modelo de Vetores Autorregressivos (VAR) no período 1980-2010.
}

\author{
Samuel Costa Peres \\ Doutorando em Economia na Universidade Federal do Rio Grande do Sul (UFRGS). \\ Endereço para contato: Rua Miguel Couto, 197 - Porto Alegre - RS \\ CEP: 90850-050 - E-mail: samuelcperes@hotmail.com
}

\section{Tiago Hideo Yamada}

Mestrando em Economia na Universidade Estadual de Maringá (UEM).

Endereço para contato: Rua Colômbia, 303 - Ibiúna - SP

CEP: 18150-000 - E-mail: hideoyamada26@gmail.com

Recebido em 04 de abril de 2014. Aceito em 12 de setembro de 2014.

\begin{abstract}
Resumo
O propósito do presente artigo é analisar os determinantes dos influxos de investimento estrangeiro direto (IED) na economia brasileira no período 1980-2010. Basicamente, os determinantes dos IEDs podem ser relativos às firmas e a características dos países de origem (push factors) ou a fatores locacionais (pull factors). Entre os fatores push analisados neste artigo, destaca-se a influência da taxa de crescimento real do PIB das economias avançadas e a taxa de variação do índice Dow Jones (DJIA). Como efeitos pull, sobressaíram-se a taxa de crescimento do produto doméstico, o Ibovespa, a taxa de câmbio e, em menor grau, o índice de Liberdade Econômica.
\end{abstract}

\section{Palavras-chave}

Fluxos de IED; modelo VAR; Brasil

\begin{abstract}
This paper aims to analyze the determinants of the foreign direct investment (FDI) inflows in the Brazilian economy over the period 1980-2010. Basically, the determinants of FDI may be related to characteristics of firms and countries of origin (push factors) or locational factors (pull factors). Among the push factors analyzed in this article, stands out the influence of the GDP real growth rate in advanced economies and the rate of change of the Dow Jones Index (DJIA). As pull effects, stood out the domestic product rate of growth, the Bovespa index, the exchange rate and, in a minor degree, the Economic Freedom Index.
\end{abstract}

\section{Keywords}

FDI flows; VAR model; Brazil

JEL Classification

F21; F41; F22. 


\section{Introdução}

A década de 1990 foi caracterizada por importantes transformações no plano econômico internacional. A globalização financeira provocou uma grande integração dos sistemas financeiros mundiais e uma expansão no movimento de capitais internacionais. Nesse período, a política macroeconômica conduzida pelas economias desenvolvidas é marcada por um afrouxamento da política monetária, o que implicou uma significativa redução das taxas de juros internacionais. Essa redução dos juros, associada à elevada liquidez internacional e a recessão que alguns países desenvolvidos atravessavam fizeram com que os mercados emergentes se constituíssem como uma alternativa para os capitais internacionais.

Na literatura sobre o tema, o aumento da participação relativa dos países em desenvolvimento no processo de globalização deve-se também a outros fatores: (i) o relativo sucesso de políticas de estabilização nessas economias em um cenário de alta liquidez internacional; (ii) a intensificação de políticas de atração de capitais por parte de países em desenvolvimento, mais abertos comercial e financeiramente; (iii) ênfase nas políticas de privatização em um número significativo de países em desenvolvimento; (iv) a crescente diversificação de posições por parte de empresas e expectativa de taxas de crescimento relativo maior nesses países.

Como aponta Lima Júnior (2005), nas economias da África e da América Latina, a média do fluxo anual de entrada de investimentos diretos foi de US\$ 63 bilhões, entre 1990 e 1994. Já no período seguinte, entre 1995 e 1998, a média desse fluxo elevou-se para US\$ 145 bilhões, isto é, o volume de investimentos diretos mais que dobrou frente ao período anterior.

No âmbito interno, no Brasil, nota-se nos anos 1990 uma intensificação dos fluxos de capitais, resultado dos esforços governamentais para inserir ativamente o país na era da financeirização ${ }^{1}$. Destaca-se o aumento expressivo dos investimentos estrangeiros diretos, sobretudo após o Plano Real. A estabilização monetária sancionou o cenário receptivo aos novos fluxos, encaminhado anteriormente pela abertura econômica e renegociação da dívida externa.

Nesse cenário, a economia brasileira surge como um dos principais países receptores do fluxo de capitais internacionais em meados da década de 1990. Dentre esses recursos externos, destaca-se o papel do investimento estrangeiro direto (IED), cujo volume, a partir da segunda metade da década, supera o volume de entrada de capitais de curto prazo, estabelecendo uma mudança significativa na estrutura de propriedade de capital no Brasil.

Os influxos de IED cada vez mais elevados na economia brasileira exerceram um papel fundamental ao longo da década de 1990, haja vista que sustentavam os volumosos déficits na conta de transações correntes e viabilizavam a valorização cambial sucedida após o Plano Real.

Basicamente, os investimentos estrangeiros diretos (IED) apontam para o controle acionário durável de empresas, sob a forma de aquisição de propriedade, fusão, joint venture ou projetos greenfield (novos investimentos). Essa modalidade de investimento é da maior importância, pois, desde que voltados para projetos greenfield (novos investimentos), representam aumento da capacidade produtiva do país que os recebe. De modo geral, o IED é

\footnotetext{
${ }^{1}$ Para uma boa reflexão sobre a inserção do Brasil na lógica da financeirização global, ver Paulani (2012).
} 
um indicador da confiança dos investidores externos na economia nacional, uma vez que expressa a disposição de arriscar capital nessa economia.

Ademais, diferentemente dos investimentos em carteira, o IED apresenta uma contrapartida de longo prazo, elevando os níveis de poupança e de investimento de um país. Ressalta-se que crescentes ingressos de IED podem também elevar a vulnerabilidade externa de uma economia, especialmente se esse processo não for acompanhado por um aumento na receita das exportações.

Ultimamente, o comportamento dos fluxos de IED é bastante complexo e relaciona-se não apenas a uma série de fatores que permeiam o ambiente competitivo em que as empresas atuam, mas também às características econômicas dos países hospedeiros e remetentes. Posto isso, compreender as razões que determinam os influxos de IED no Brasil é um campo de estudo relevante.

Nesse sentido, o propósito deste artigo é verificar se alguns fatores push e pull são relevantes para explicar o crescimento dos fluxos de IED no Brasil no período 1980-2010. Para tal intento, será utilizado o modelo de vetores autoregressivos (VAR), e as variáveis utilizadas são: ingressos líquidos de IED; taxa de crescimento real do Produto Interno Bruto (PIB) do Brasil; taxa de crescimento real do PIB das economias avançadas ${ }^{2}$; taxa de juros nominal; taxa de câmbio nominal; inflação (IPC); índice Bovespa; índice Dow Jones; Índice de Liberdade Econômica.

Além dessa introdução, o artigo está divido em mais cinco seções. Na primeira seção é apresentada a evolução do IED na economia brasileira no período de 1980 a 2010. A segunda seção reserva-se a uma revisão da literatura empírica sobre os determinantes do IED nos países em desenvolvimento, entre eles, o Brasil. A terceira parte descreve a metodologia a ser aplicada no trabalho e, na quarta, são apresentados e discutidos os resultados do modelo. Na quinta e última seção são apresentadas as considerações finais.

\section{Evolução do IED no Brasil: 1980-2010}

Na América Latina, como se sabe, a década de 1980 é marcada pela crise da dívida externa, que abalou o cenário econômico e o crescimento dos países da região e, consequentemente, comprometeu o financiamento externo dessas economias, entre elas, o Brasil.

No contexto de intensificação da globalização econômica, o processo de reestruturação da indústria brasileira, iniciado a partir dos anos 1990, trouxe consigo um fluxo expressivo de investimento estrangeiro direto, transformando o país em um dos principais polos de atração desse capital.

Pode-se dizer que o país se inseriu no processo de globalização por meio da adoção de um conjunto de medidas liberalizantes, com intuito de promover a abertura comercial e a desregulamentação do mercado interno. Ou seja, o Brasil adotou um programa de estabilização apoiado em uma estratégia de abertura financeira, em que foram retirados todos os obstáculos a livre movimentação dos fluxos de capitais, com a desregulamentação do mercado financeiro (JABBOUR; ACIOLY; MIGUEL, 2006).

\footnotetext{
${ }^{2}$ Constituem o grupo das economias avançadas os países do G7, da zona do Euro e as economias asiáticas recentemente industrializadas (Hong Kong, Coréia do Sul, Cingapura e Taiwan).
} 
No gráfico 1, nota-se que os fluxos de IED intensificam-se na década de 1990, mais precisamente a partir de 1994, sendo no período anterior pouco significativos, em termos relativos. A tendência declinante dos investimentos diretos de 2000 a 2004 deve-se em boa parte ao excesso de atenção ao controle da inflação com a manutenção das taxas de juros muito elevadas, à falta de investimentos do setor público em setores estratégicos, à alta carga tributária, além da indefinição com respeito à atuação das agências reguladoras. Para Costa, 2002, internamente, deve-se ressaltar também a diminuição das privatizações e das fusões e aquisições. IDE No âmbito externo, soma-se a isso a desaceleração da economia mundial, o baixo crescimento econômico e a recessão nos Estados Unidos. Em 2004, esse movimento se reverte, possivelmente em decorrência da alta no preço das commodities, que acabou atraindo mais IED para setores ligados aos recursos naturais e agrícolas como energia, extrativo mineral, agropecuária, metalurgia e siderurgia, indústria de alimentos, etc.

No que tange à grande queda registrada no saldo em 2006, esta se deve ao aumento abrupto dos investimentos brasileiros diretos, dos quais U\$ 24,41 bilhões tiveram como destino a participação no capital de empresas estrangeiras. Por outro lado, em 2007, houve uma grande elevação dos recursos no mercado financeiro brasileiro, e tanto os investimentos diretos quanto os em carteira tiveram forte participação. Esse resultado parece estar associado, entre outros fatores, à queda do risco-brasil, à boa remuneração dos títulos que atraem compradores estrangeiros, ao crescimento do mercado acionário devido principalmente ao bom desempenho das grandes empresas nacionais, e ao aumento da demanda interna com a popularização do crédito, que criou oportunidade para diversos setores da economia.

\section{Gráfico 1 - Fluxos de IED no Brasil: 1980-2010 (US\$ bilhões)}

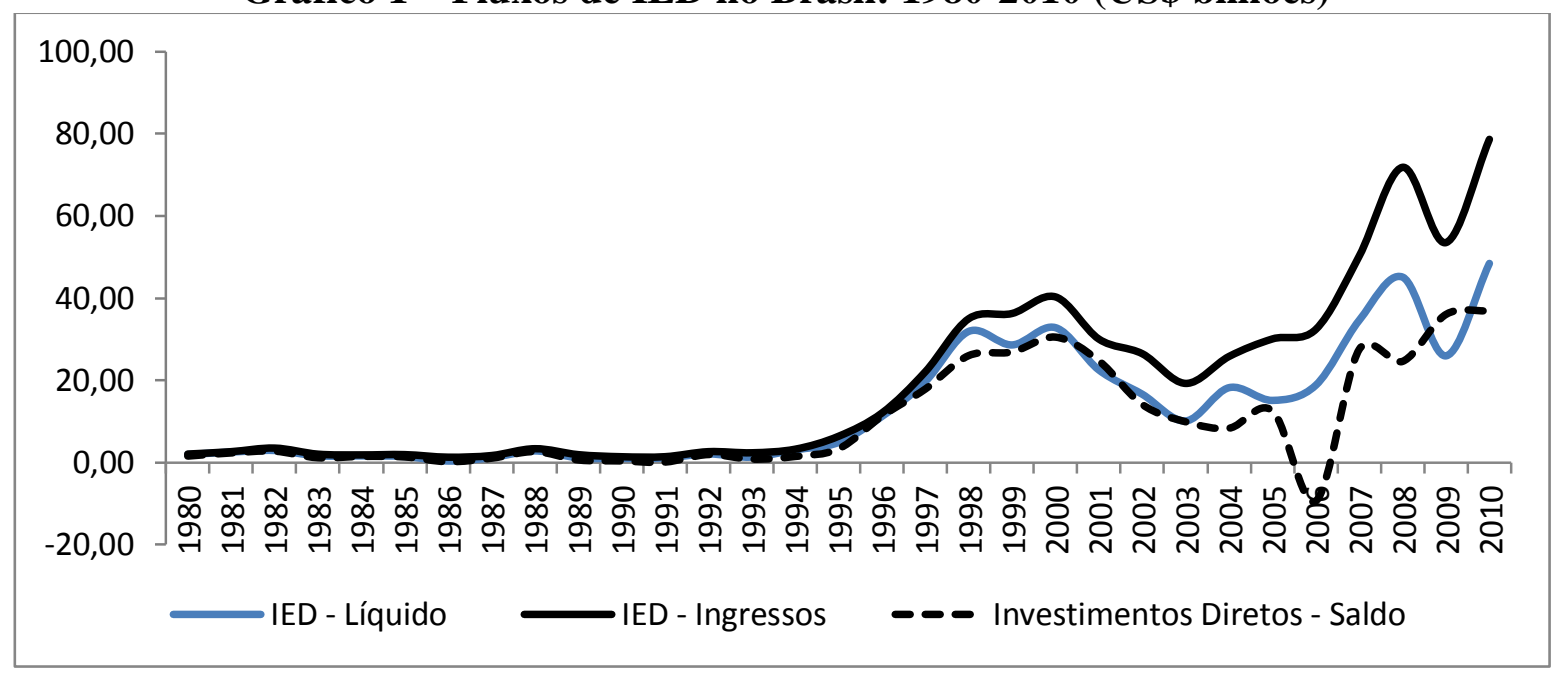

Fonte: Ipeadata. Elaboração própria.

O crescente fluxo de capitais externos para o mercado financeiro brasileiro manteve-se até meados do segundo semestre de 2008. Contudo, refletindo a crise financeira internacional, houve uma forte queda nos influxos de IED não apenas na economia brasileira, mas na maioria dos emergentes. Vale mencionar que, nesse cenário de crise, o saldo dos investimentos diretos não apresentou queda mais drástica devido à grande repatriação das inversões brasileiras no exterior. Não obstante, em 2010 retomam-se os investimentos 
estrangeiros no país, com o fluxo líquido de IED atingindo o valor recorde de US\$ 48,4 bilhões, sendo o valor bruto correspondente a US\$ 78,6 bilhões.

\section{Evidências empíricas sobre os determinantes do investimento estrangeiro direto nos países em desenvolvimento}

Majoritariamente, a literatura teórica relativa ao investimento direto estrangeiro e às empresas multinacionais tem se concentrado na análise nas firmas, mais especificamente, quais deveriam ser as vantagens internas às firmas que as possibilitariam inserir suas atividades no mercado internacional, e em condições de competir com as firmas domésticas. Nessa literatura, destacam-se os trabalhos de Hymer (1960, 1968, 1976), centrados na organização industrial e nas imperfeições de mercado, o Ciclo do Produto de Vernon (1966) associado à internacionalização da produção, Buckey e Casson (1976; 1981), cujo foco é a internalização dos custos, além do Paradigma Eclético de Dunning (1988, 1993, 2001). Nessas abordagens, no entanto, menor importância foi dada aos fatores macroeconômicos.

$\mathrm{Na}$ literatura empírica sobre o tema, por sua vez, diversos trabalhos têm centralizado o foco nos determinantes macroeconômicos dos fluxos de IED, sobretudo para os países em desenvolvimento. Os trabalhos empíricos que buscam estimar a importância dos determinantes dos fluxos de IED têm enfatizado mais os fatores de atração, isto é, os fatores locacionais, uma vez que os dados raramente possibilitam identificar os países de origem dos investimentos para um grande número de países e anos. Com isso, as variáveis comumente usadas são o tamanho do mercado, a taxa de crescimento do PIB, a estabilidade econômica, o grau de abertura, entre outras de natureza institucional ${ }^{3}$.

No que se refere aos trabalhos voltados aos países em desenvolvimento em geral, destacam-se algumas dessas contribuições.

Schneider e Frey (1985) analisaram os fatores determinantes do fluxo líquido de investimento direto externo para um total de 54 países da América Latina, Ásia e África, utilizando regressão múltipla para os anos de 1976, 1979 e 1980. Utilizando-se de variáveis econômicas e políticas, os resultados revelaram que, na esfera econômica, quanto maior o PIB per capita e quanto menor o déficit no balanço de pagamentos maior a atração de IED. No campo político, quanto maior a assistência dos países ocidentais maior o estímulo ao investimento estrangeiro e quanto maior a instabilidade política (uma dummy representando a ideologia do governo) menor o influxo de IED.

Holland et al (2000), em um levantamento de diversos trabalhos empíricos focalizando a Europa Oriental e Central mostram evidências de associação econométrica entre tamanho do mercado e potencial de crescimento com fluxos de IED. Lane e Milesi-Ferretti (2000) estimaram um modelo de determinação de estoque de passivos externos para 132 países em 1997, dividindo-os em economias industriais e em desenvolvimento, e desagregando os fluxos externos em IED, investimentos em carteira e empréstimos. No que tange aos determinantes do IED, foram observadas disparidades entre os dois grupos de países. No caso

\footnotetext{
${ }^{3}$ Ressalta-se que a possibilidade de haver problemas de endogeneidade na relação entre IED e crescimento do produto deve ser avaliada. Se, por um lado, os fluxos de IED tendem a aumentar a taxa de crescimento do produto em etapa posterior ao ingresso, uma vez que representam expansão bruta do estoque de capital, de outro, a própria dinâmica do produto pode influenciar a captação de IED.
} 
dos países industriais, a renda per capita, o grau de abertura comercial e a capitalização do mercado de capitais em relação ao Produto Nacional Bruto (PNB) foram as variáveis que explicaram a maior parte dos passivos em IED. Para as economias em desenvolvimento, por sua vez, as variáveis significativas e positivamente correlacionadas com as obrigações de IED foram a parcela dos recursos naturais exportáveis e das receitas das privatizações relativamente ao PNB, e a abertura comercial.

Garibaldi et al (2001), por meio de um painel dinâmico com 26 países em transição entre 1990-1999, encontraram dados significativos na explicação dos fluxos de IED para as seguintes variáveis macroeconômicas: tamanho do mercado, déficit fiscal, inflação e regime cambial, além de percepção de risco, reformas econômicas, liberalização comercial, dotação de recursos naturais, barreiras ao investimento e burocracia.

Utilizando uma amostra de 28 países em desenvolvimento para o período 1987-2000, Nunnenkamp e Spatz (2002), encontraram correlações de Spearman significativas entre fluxos de IED e PIB per capita, fatores de risco, anos de escolaridade, restrições ao comércio externo, fatores complementares de produção, gargalos administrativos e fatores de custo. Opostamente, crescimento do PIB, população, restrições à entrada de firmas, restrições após a entrada e regulação relacionada com tecnologia revelaram-se não significativas.

Valendo-se da análise em painel, Campos e Kinoshita (2003) analisaram 25 economias em transição entre 1990 e 1998, tendo encontrado evidências de que o IED é influenciado por clusters, tamanho do mercado, recursos naturais abundantes e baixo custo da mão de obra, abertura comercial e menores restrições aos fluxos de IED, além de boas instituições.

No intuito de avaliar a efetividade de alguns fatores macroeconômicos na atração de investimentos estrangeiro diretos, Nonnenberg e Mendonça (2004) utilizaram um painel de dados incluindo 33 países em desenvolvimento no período de 1975 a 2000. Os resultados evidenciaram que fatores como tamanho e ritmo de crescimento do produto, qualificação da mão-de-obra, risco-país, desempenho do mercado de capitais e grau de abertura da economia estão entre os principais determinantes de IED, apresentando os sinais esperados e sendo significativos. Ademais, a partir de um teste de causalidade, os autores verificaram que essa modalidade de investimentos não possui efeito sobre o PIB do país receptor, e ao contrário, é o PIB que afeta a entrada desses investimentos.

Em relação a trabalhos empíricos específicos para o Brasil, pode-se destacar Costa (2002), que utilizou um modelo de dados em painel para identificar as variáveis mais significativas para os influxos de IED no Brasil. Os resultados apontaram que na década de 1990, a dimensão do mercado interno foi decisiva para a entrada desses capitais. Variáveis como o PIB do Brasil e dos países de origem dos investimentos também se mostraram estatisticamente significativas, além da taxa de câmbio, dos custos salariais, das privatizações, da distância geográfica e da dotação de recursos naturais.

Outro estudo, realizado por Mattos et al. (2007), analisa como o ingresso de IED no Brasil respondeu às mudanças nos níveis dos determinantes principais sugeridos pela literatura teórica e empírica sobre o tema. Os autores estimaram um modelo de Correção de Erro Vetorial (VEC) para o período 1980-2004. As conclusões foram que o IED foi mais sensível ao risco país, ao grau de abertura comercial e à taxa de inflação doméstica. Por outro lado, mudanças na taxa de crescimento do PIB e na taxa de câmbio apresentaram impactos menores. 
Ainda no que se refere ao Brasil, Lima Júnior (2005), a partir de uma amostra com 49 setores no período 1996-2003, evidencia que o principal determinante dos influxos de IED foi a busca por novos mercados, uma vez que o tamanho do mercado interno, representado pelo PIB, e seu histórico de crescimento afetaram positivamente as entradas desses capitais. Ademais, o coeficiente de abertura comercial, a taxa de inflação, o desempenho das bolsas de valores e o estoque de IED foram estatisticamente significativos.

\section{Investigação econométrica: modelos VAR}

À luz dos trabalhos empíricos apresentados na seção anterior, o objetivo desta seção é analisar os determinantes dos influxos de IED no Brasil por meio da estimação de modelos recursivos de Vetores Autoregressivos (VAR) e do instrumental da Análise de Decomposição de Variância (ADV) e do Impulso-resposta, para o período 1980-2010. Nesse sentido, a subseção que segue reserva-se aos fundamentos teóricos do modelo utilizado no trabalho. A segunda subseção, então, apresenta as variáveis escolhidas, bem como suas fontes e os modelos a serem estimados. Por fim, são apresentados e discutidos os resultados subjacentes ao objetivo aqui proposto.

\subsection{Fundamentos do modelo VAR}

O estudo das inter-relações entre variáveis econômicas, em modelos de regressão múltiplas, é realizado pela técnica de equações simultâneas. Nesses modelos, as variáveis são classificadas como endógenas e exógenas (pré-determinadas) e para sua estimação é preciso alguma restrição. Para poder estimar um modelo de equação simultânea é necessário saber se ele é identificado, exatamente identificado ou sobreidentificado e, a partir daí, é possível saber se o sistema possui solução.

Nos modelos de equações simultâneas o problema da identificação é resolvido incorporando-se variáveis pré-determinadas em algumas equações e em outras não. Por essa razão, Sims (1980) defendeu a ideia de que todas as variáveis devem ser tratadas de forma simultânea e simétrica. A partir dessa discussão, houve a introdução de uma nova abordagem de séries multivariadas: o modelo de Vetor Autorregressivo.

Considerando-se por simplificação o caso de duas variáveis, pode-se analisar como a trajetória de tempo de uma variável $\left(Y_{t}\right)$ pode ser afetada pelas realizações correntes e passadas de outra variável $\left(Z_{t}\right)$, e vice-versa. O modelo primitivo (ou VAR estrutural), com $i$ defasagens, pode ser escrito na seguinte forma:

$$
\begin{aligned}
& Y_{t}=b_{10}-b_{12} Z_{t}+\delta_{12} Y_{t-i}+\delta_{12} Z_{t-i}+\varepsilon_{y t} \\
& Z_{t}=b_{20}-b_{21} Y_{t}+\delta_{21} Y_{t-i}+\delta_{22} Z_{t-i}+\varepsilon_{z t}, t=0,1,2 \ldots n
\end{aligned}
$$

onde: (i) ambas as variáveis apresentam as propriedades do modelo de Box-Jenkins (parcimônia, invertibilidade e estabilidade) e (ii) $\varepsilon_{y t}$ e $\varepsilon_{z t}$ são distúrbios de ruído branco não correlacionados com desvio padrão de $\sigma_{y}$ e $\sigma_{z}$, respectivamente.

A estrutura do sistema indica relações simultâneas entre $Y_{t}$ e $Z_{t}$ e os distúrbios $\varepsilon_{y t} \mathrm{e}$ $\varepsilon_{z t}$ tem um efeito indireto contemporâneo sobre $Y_{t}$ e $Z_{t}$, respectivamente. Esse fato torna os 
choques relacionados com as variáveis explicativas, violando assim, um dos pressupostos do modelo de Mínimos Quadrados Ordinários que é o de não correlação entre os regressores e o termo de erro. Essa violação não permite a estimação por esse método.

Dessa forma, para estimar o sistema primitivo (equações 1. $a$ e 1.b) pelo método de Mínimos Quadrados Ordinários é necessário transformá-lo na forma reduzida. Aplicando-se a álgebra de matrizes, o sistema pode ser escrito como:

Onde:

$$
B \times X_{t}=\Gamma_{0}+\Gamma_{1} X_{t-i}+\varepsilon_{t}
$$

$$
B=\left[\begin{array}{cc}
1 & b_{12} \\
b_{21} & 1
\end{array}\right], \quad X_{t}=\left[\begin{array}{l}
Y_{t} \\
Z_{t}
\end{array}\right], \quad \Gamma_{0}=\left[\begin{array}{l}
b_{10} \\
b_{20}
\end{array}\right], \quad \Gamma_{1}=\left[\begin{array}{ll}
\delta_{11} & \delta_{12} \\
\delta_{21} & \delta_{22}
\end{array}\right], \quad X_{t-i}=\left[\begin{array}{c}
Y_{t-i} \\
Z_{t-i}
\end{array}\right], \quad \varepsilon_{t}=\left[\begin{array}{c}
\varepsilon_{y t} \\
\varepsilon_{z t}
\end{array}\right]
$$

Pré-multiplicando a equação (2) por uma matriz inversa $\left(B^{-1}\right)$, obtêm-se o modelo VAR padrão:

$$
X_{t}=A_{0}+A_{1} X_{t-i}+e_{t}
$$

$X_{t}$ corresponde a um vetor $(n x 1)$ contendo $n$ variáveis incluídas no VAR; $A_{0}$ refere-se ao vetor $(n \times 1)$ de interceptos; $A_{1}$ é igual à matriz $(n \times n)$ de coeficientes e $e_{t}$ é o vetor $(n \times 1)$ de termos de erros.

Solucionado o problema do sistema de equações, o instrumento essencial para analisar a dinâmica da inter-relação entre as variáveis em um VAR é a representação deste em um vetor de médias móveis (VMA). Tal representação é possível desde que sejam respeitadas as condições de estacionariedade e invertibilidade do modelo de Box-Jenkins (ENDERS, 1995).

A representação VMA da equação (3) expressa as variáveis $Y_{t}$ e $Z_{t}$ em termos dos valores correntes e passados de dois choques $\varepsilon_{y t}$ e $\varepsilon_{z t}$. Considerando-se a expressão em termos de choques e realizando-se algumas operações algébricas, obtêm-se:

$$
X_{t}=\mu+\sum_{0}^{\infty} \varphi_{i} \varepsilon_{t-1}
$$

Os coeficientes de $\varphi_{i}$ são chamados de Função de Resposta a Impulsos, ou seja, mede o impacto nas variáveis dado o impulso (choques) nos resíduos $\varepsilon_{y t}$ e $\varepsilon_{z t}$.

Entretanto, o modelo na forma reduzida não permite a identificação dos efeitos dos choques exógenos independentes nas variáveis. Os erros do modelo VAR restrito $\left(\varepsilon_{y t}, \varepsilon_{z t}\right)$, são compostos por combinações dos choques estruturais, isto é, podem ser correlacionados contemporaneamente. Dessa forma, só é possível fazer a identificação do choque em uma única variável do sistema impondo restrições sobre as relações contemporâneas das variáveis. Uma possível identificação restrita pode ser feita utilizando os modelos recursivos, que são construídos de modo que os termos de erros de cada equação sejam não correlacionados com os erros da equação precedente. Nesses modelos, o método mais amplamente utilizado é a denominada Decomposição de Choleski, em que $Y_{t}$ não tem efeito contemporâneo sobre $Z_{t}$. Em termos formais, esta restrição é representada fixando $b_{21}=0$ no sistema primitivo.

Aplicando o método de Choleski para $n$ variáveis, a ordenação do VAR pode alterar o resultado, pois a variável que ocupa a primeira posição tem efeitos contemporâneos sobre as 
outras variáveis do sistema, enquanto que a variável que ocupa a última posição não exerce efeitos contemporaneamente às outras variáveis, sendo influenciada por todas as outras variáveis (Janeiro, 2004).

É importante observar que devido ao fato das variáveis inseridas no sistema VAR serem em muitos casos relacionadas, pode-se utilizar a análise de Decomposição de Variância do Erro (ADV) para mostrar qual impacto cada variável apresenta na previsão da variância do erro-padrão da outra série. Essa análise possibilita examinar se existe alguma série que pode ser considerada exógena ao sistema, ou seja, se esta sofre um impacto somente do passado dela mesma e qual a importância da sua defasagem para a estimação do modelo.

\subsection{As variáveis, fontes de dados e os modelos}

A variável dependente, ou seja, a ser explicada neste trabalho é o fluxo de IED líquido, nominado ied_líq, e as variáveis explicativas são taxa de crescimento real do PIB do Brasil (tcpibbrasil), taxa de crescimento real do PIB das economias avançadas (tcpibadvecon), taxa de câmbio nominal (tcambio), taxa de juros de curto prazo nominal, a Selic (juros), taxa de inflação, sendo esta o índice de preços ao consumidor (inf_CPI), a variação percentual do índice dos principais papéis negociados na BMF\&Bovespa, a bolsa de valores de São Paulo (ibov_per), a variação percentual do índice das 30 maiores empresas dos EUA cotadas na Bolsa de Valores de Nova Iorque ${ }^{4}$, o Dow Jones Industrial Average (DJIA), denominado aqui por djia_per e, por fim, o Índice de Liberdade Econômica (Economic Freedom Index), nominado EFI.

As variáveis ied_liq, tcambio, juros, inf_CPI foram extraídas do banco de dados do IPEA, o Ipeadata. As variáveis tcpibbrasil e tcpibadvecon foram extraídas do banco de dados do Fundo Monetário Internacional (FMI); ibov_per e djia_per, por sua vez, foram coletadas pelo software de informações financeiras da Agência Estado, o AE Brodcast. O EFI foi extraído da organização canadense de pesquisa e educação, o The Fraser Institute, que no cálculo do índice de liberdade econômica considera 38 componentes e subcomponentes de 5 grandes áreas: (i) tamanho do governo; (ii) estrutura legal e proteção ao direito de propriedade; (iii) acesso a moeda estável, (iv) intercâmbio com o estrangeiro e; (v) regulamentação.

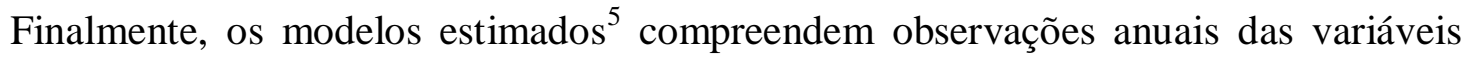
supracitadas, abarcando o período 1980-2010. Em decorrência do número de variáveis e observações, optou-se pela estimação de dois modelos separadamente, como segue:

1. Modelo I: IED, TCPIBBRASIL, TCPIBADVECON, JUROS, TCAMBIO, INF_CPI

2. Modelo II: IED, DJIA_PER, IBOV_PER, EFI

A sequência das variáveis foi escolhida conforme a ordenação proposta por Choleski, em que, conforme já mencionado, a variável que ocupa a primeira posição tem efeitos

\footnotetext{
${ }^{4}$ Em 1999, Intel Corp. e Microsoft começaram a compor o índice e se tornaram as primeiras duas empresas negociadas na NASDAQ a participarem da composição do DJIA. Todas as outras empresas têm suas ações negociadas na Bolsa de Valores de Nova Iorque (New York Stock Exchange).

${ }^{5} \mathrm{Na}$ estimação dos modelos VAR utilizou-se o programa econométrico STATA 12.
} 
contemporâneos sobre as outras variáveis do sistema, enquanto que a variável que ocupa a última posição não exerce efeitos contemporaneamente às outras variáveis, sendo influenciada por todas as outras variáveis.

\section{Apresentação e discussão dos resultados}

\subsection{Testes de raiz unitária}

Antes de se estimar um modelo VAR, o primeiro procedimento é verificar se as séries são estacionárias, e para isso utiliza-se o conceito de Raiz Unitária ${ }^{6}$. Segundo Nelson e Plosser (1982), o principal problema econômico resultante da presença de raiz unitária em uma série temporal reside no fato de que choques aleatórios geram efeitos permanentes sobre a variável, tornando as flutuações destas não transitórias.

Para avaliar se as variáveis seguem um padrão estocástico estacionário, foram realizados três testes de raiz unitária. A figura 3.1 sintetiza os resultados dos testes de DickeyFuller Aumentado (ADF), Dickey-Fuller GLS (ADF-GLS), de Elliott, Rothenberg e Stock, 1996, e Phillips-Perron (PP) de 1987. Os asteriscos mostram o nível de significância de cada teste para a variável em questão. As hipóteses nulas dos testes são de que as séries são não estacionárias.

Para o teste Dickey-Fuller Aumentado, as variáveis tcpibbrasil, tcpibadvecon, ibov_per e djia_per apresentaram-se estacionárias em nível com um nível de significância de $1 \%$, já as variáveis ied_li, juros, inf_CPI, EFI mostraram-se estacionárias em $1^{\mathrm{a}}$ diferença com $1 \%$ de significância, a variável tcambio mostrou-se estacionária a 5\% de significância.

O teste Dickey-Fuller GLS apresentou resultados parecidos com o teste ADF, com exceção tcpibbrasil que não se mostrou estacionária a nenhum nível de significância, e da variável EFI que se mostrou estacionária em $1^{\mathrm{a}}$ diferença só que a um nível de significância de $10 \%$.

O teste Phillips-Perron exibiu os mesmo resultados do teste Dickey-Fuller Aumentado. Apesar da divergência do teste Dickey-Fuller GLS da variável tcpibbrasil em relação ao resultado dos outros dois testes decidiu-se desconsiderar o resultado do teste Dickey-Fuller GLS, e assim a variáveis tcpibbrasil, tcpibadvecon, ibov_per e djia_per serão tratadas em nível, enquanto que as variáveis ied_li, juros, inf_CPI, tcambio, EFI serão tratadas em primeira diferença conforme a tabela 1.

Tabela 1 - Testes de Raíz Unitária

\begin{tabular}{c|lcccc}
\hline Modelo & \multicolumn{1}{|c}{ Variável } & ADF & ADF-GLS & PP & Decisão \\
\hline \multirow{6}{*}{$\mathbf{1}$} & IED_liq $^{1}$ & $\mathrm{I}(1)^{* * *}$ & $\mathrm{I}(1)^{* * *}$ & $\mathrm{I}(1)^{* * *}$ & $\mathrm{I}(1)$ \\
& tcpibbrasil $^{2}$ & $\mathrm{I}(0)^{* * *}$ & - & $\mathrm{I}(0)^{* * *}$ & $\mathrm{I}(0)$ \\
& tcpibadvecon $^{2}$ & $\mathrm{I}(0)^{* * *}$ & $\mathrm{I}(0)^{* * *}$ & $\mathrm{I}(0)^{* * *}$ & $\mathrm{I}(0)$ \\
& juros $^{2}$ & $\mathrm{I}(1)^{* * *}$ & $\mathrm{I}(1)^{* * *}$ & $\mathrm{I}(1)^{* * *}$ & $\mathrm{I}(1)$ \\
& tcambio $^{2}$ & $\mathrm{I}(1)^{* *}$ & $\mathrm{I}(0)^{* *}$ & $\mathrm{I}(1)^{* * *}$ & $\mathrm{I}(1)$ \\
& inf_CPI $^{2}$ & $\mathrm{I}(1)^{* * *}$ & $\mathrm{I}(1)^{* * *}$ & $\mathrm{I}(1)^{* * *}$ & $\mathrm{I}(1)$ \\
\hline
\end{tabular}

${ }^{6}$ Vide Nelson e Plosser(1982), Bueno(2011, cap.4), Greene (2011, cap.21) 


\begin{tabular}{c|lcccc}
2 & IED_liq & $\mathrm{I}(1)^{* * *}$ & $\mathrm{I}(1)^{* * *}$ & $\mathrm{I}(1)^{* * *}$ & $\mathrm{I}(1)$ \\
& djia_per $^{2}$ & $\mathrm{I}(0)^{* * *}$ & $\mathrm{I}(0)^{* * *}$ & $\mathrm{I}(0)^{* * *}$ & $\mathrm{I}(0)$ \\
& ibov_per $^{2}$ & $\mathrm{I}(0)^{* * *}$ & $\mathrm{I}(0)^{* * *}$ & $\mathrm{I}(0)^{* * *}$ & $\mathrm{I}(0)$ \\
& EFI $^{2}$ & $\mathrm{I}(1)^{* * *}$ & $\mathrm{I}(1)^{*}$ & $\mathrm{I}(1)^{* * *}$ & $\mathrm{I}(1)$ \\
\hline
\end{tabular}

Fonte: Stata12. Elaboração própria.

( - ) - Sem resultados significativos .

${ }^{1}$ Tendência e Intercepto, ${ }^{2}$ Intercepto.

$*$ significativo a $10 \%, * *$ significativo a $5 \%, * * *$ significativo a $1 \%$.

\subsection{Seleção de defasagens e testes de robustez}

Para determinar o número de defasagens das variáveis a serem incluídas no modelo, foram realizados os usuais testes de seleção do número de defasagens do sistema VAR. A tabela 2 mostra os resultados para os modelos I e II.

Para o modelo I, a estatística LR, os critérios de AIC, SC e HQ sugerem a inclusão de seis defasagens ao modelo, enquanto o critério de FPE sugere a inclusão de três. No modelo II, a estatística LR, os critérios de AIC, SC e HQ também sugerem a inclusão de seis defasagens ao modelo, enquanto o FPE sugere cinco.

As estimativas dos VARs com 6 defasagens para ambos os modelos resultou em modelos cujos resíduos estavam autocorrelacionados, heterocedásticos e não-gausianos, ou seja, o modelo não mostrou-se estável. Ressalta-se ainda a impossibilidade, em termos de confiabilidade estatística, de se ter um número de variáveis superior ao número de observações, motivo pelo qual se deve impor limites ao número de lags.

Para resolver tal problema foi escolhido um número menor de defasagens, em que foi possível estimar os modelos com resíduos bem comportados, e estáveis. Ao final, optou-se por estimar um VAR (3) para ambos os modelos, satisfazendo as condições básicas de robustez, como será apresentado a seguir.

Tabela 2 - Seleção de defasagens (VAR)

\begin{tabular}{ccccccc}
\hline \multicolumn{7}{c}{ Modelo I } \\
\hline lag & LogL & LR & FPE & AIC & SC & HQ \\
\hline 0 & -485.157 &. & $1.40 \mathrm{E}+11$ & 42.7093 & 42.7838 & 43.0055 \\
1 & -441.149 & 88.017 & $8.00 \mathrm{E}+10$ & 42.0129 & 42.5344 & 44.0864 \\
2 & -385.391 & 111.52 & $3.10 \mathrm{E}+10$ & 40.2949 & 41.2633 & 44.1457 \\
3 &. &. & $-4.1 \mathrm{e}-20^{*}$ &. &. &. \\
4 & 3992.3 &. &. & -335.156 & -333.443 & -328.343 \\
5 & 4006.88 & 29.173 &. & -336.425 & -334.711 & -329.612 \\
6 & 4049.74 & $85.717^{*}$ &. & $-340.152^{*}$ & $-338.438^{*}$ & $-333.339^{*}$ \\
\hline
\end{tabular}

Modelo II

\begin{tabular}{ccccccc}
\hline lag & LogL & LR & FPE & AIC & SC & HQ \\
\hline 0 & -211.61 &. & 747.082 & 17.9675 & 18.0196 & 18.1638 \\
1 & -198.174 & 26.871 & 948.083 & 18.1812 & 18.4416 & 19.1629 \\
2 & -191.931 & 12.487 & 2432.23 & 18.9942 & 19.463 & 20.7613 \\
3 & -181.148 & 21.566 & 5411.34 & 19.429 & 20.1062 & 21.9815 \\
4 & -163.721 & 34.854 & 11644.4 & 19.3101 & 20.1956 & 22.6479
\end{tabular}




\begin{tabular}{ccccccc}
5 & 346.875 & 1021.2 & $1.7 \mathrm{e}-13^{*}$ & -21.9063 & -20.8124 & -17.7831 \\
6 & 3019.02 & $5344.3^{*}$ &. & $-243.585^{*}$ & $-242.335^{*}$ & $-238.873^{*}$ \\
\hline
\end{tabular}

Fonte: elaboração própria. (*) Indica o número de defasgens selecionado por cada critério. LR: estatística LR; FPE: erro final de previsão; AIC: critério de informação Akaike; SC: critério de informação Schwarz; HQ: critério de informação de Hannan-Quinn.

Em relação aos testes de robustez, realizou-se o diagnóstico dos resíduos por meio do teste de normalidade de Jarque-Bera e do teste do Multiplicador de Lagrange (LM) para autocorrelação, bem como a verificação da estabilidade dos modelos nas correspondentes defasagens. A tabela 3 ilustra os resultados do teste de normalidade de Jarque-Bera para os modelos I e II.

\begin{tabular}{ccccc}
\multicolumn{4}{c}{ Tabela 3 - Teste de Normalidade - Jarque-Bera } \\
\hline \multirow{2}{*}{ Modelo } & Equation & Chi-sq & df & Prob. \\
& & & & \\
\hline \multirow{4}{*}{ I } & died_liq & 0.347 & 2 & 0.84086 \\
& tcpibbrasil & 1.56 & 2 & 0.4584 \\
& tcpibadvecon & 0.01 & 2 & 0.99478 \\
& djuros & 1.218 & 2 & 0.54398 \\
& dtcambio & 1.176 & 2 & 0.5554 \\
& dinf_CPI & 2.009 & 2 & 0.36624 \\
& ALL & 6.32 & 12 & 0.89911 \\
\hline \multirow{4}{*}{ II } & died_liq & 0.430 & 2 & 0.8066 \\
& djia_per & 0.349 & 2 & 0.83997 \\
& ibov_per & 0.274 & 2 & 0.87209 \\
& dEFI & 5.821 & 2 & 0.05445 \\
& ALL & 6.873 & 8 & 0.55037 \\
\hline
\end{tabular}

Fonte: Stata12. Elaboração própria.

Conforme a tabela 3, o teste de normalidade de Jarque-Bera evidencia a não-rejeição da hipótese nula de que os erros em conjunto (ALL) seguem uma distribuição normal, de modo que pode-se admitir que os resíduos dos dois modelos seguem uma distribuição próxima da normal.

Prosseguindo com o diagnóstico dos resíduos empregou-se o teste LM para autocorrelação, cujos resultados estão sistematizados na tabela 4:

Tabela 4 - Teste de Autocorrelação Serial - LM

\begin{tabular}{ccccc}
\hline Modelo & Lag & Chi-sq & df & Prob. \\
\hline \multirow{4}{*}{ I } & 1 & 37.9673 & 36 & 0.37978 \\
& 2 & 27.4667 & 36 & 0.84566 \\
& 3 & 29.3201 & 36 & 0.77698 \\
& 4 & 58.9396 & 36 & 0.00929 \\
& 5 & 43.7468 & 36 & 0.17567 \\
\hline \multirow{4}{*}{ II } & 1 & 15.8998 & 16 & 0.45997 \\
& 2 & 9.9068 & 16 & 0.87145 \\
& 3 & 22.7169 & 16 & 0.12151 \\
& 4 & 16.2462 & 16 & 0.43591 \\
& 5 & 13.7211 & 16 & 0.61948 \\
\hline
\end{tabular}

Fonte: Stata12. Elaboração própria. 
De acordo com os resultados da tabela 4, não se pode rejeitar a hipótese nula (ausência de autocorrelação serial) para a terceira defasagem, isto é, há forte evidência que, ao número de lags selecionados para os dois modelos não há o problema de autocorrelação serial entre os resíduos.

Por fim, testou-se a estabilidade dos modelos. Conforme as Figuras 1 e 2, todas as raízes do polinômio característico pertencem ao círculo unitário, comprovando, assim, a estabilidade do sistemas VARs.

\section{Figura 1 - Raízes do Polinômio Característico - Modelos I e II}
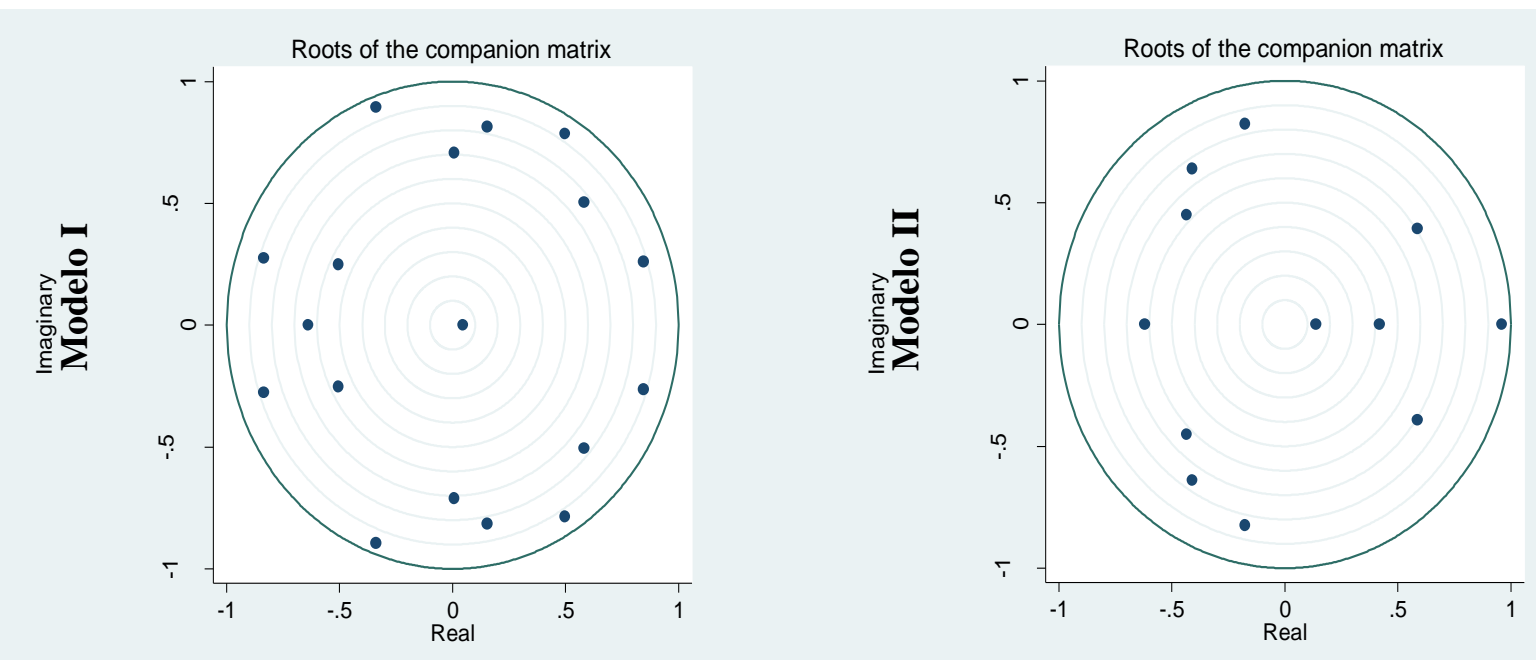

Fonte: Stata12.

\subsection{Teste de Causalidade de Granger}

O teste de causalidade de Granger indica se, dados os valores passados de duas variáveis, uma ajuda a prever a outra (variável dependente), isto é, se determinada variável causa, no sentido de Granger, a outra. Esse teste oferece também um subsídio para identificar a precedência temporal entre variáveis. Na tabela 5 estão representados os resultados do teste de causalidade de Granger para as variáveis dos modelos.

\section{Tabela 5 - Teste de Causalidade de Granger}

\begin{tabular}{cccccc}
\hline \multirow{2}{*}{ Modelo } & \multirow{2}{*}{ Variável dependente } & Excluida & Chi-sq & df & Prob. \\
\hline \multirow{4}{*}{ I } & tcpibbrasil & 7.8019 & 3 & 0.050 \\
& \multirow{3}{*}{ died_liq } & tcpibadvecon & 9.4684 & 3 & 0.024 \\
& & djuros & 7.8021 & 3 & 0.050 \\
& dtcambio & 20.027 & 3 & 0.000 \\
& dinf_CPI & 4.6369 & 3 & 0.200 \\
& ALL & 39.198 & 15 & 0.001 \\
\hline \multirow{3}{*}{ II } & dijia_per & 7.8287 & 3 & 0.050 \\
& \multirow{2}{*}{ died_liq } & ibov_per & 4.6787 & 3 & 0.197 \\
& & dEFI & 3.9006 & 3 & 0.272 \\
& & ALL & 17.983 & 9 & 0.035 \\
\hline
\end{tabular}

Fonte: Stata12. Elaboração própria. 
No caso do modelo I, rejeita-se a hipótese de que as variáveis tcpibbrasil, tcpibadvecon, juros e cambio, e todas em conjunto, não causam (no sentido de Granger), ied_liq. No modelo II, pode-se rejeitar a hipótese de que djia_per e todas as variáveis em conjunto, não causam (no sentido de Granger) ied_liq.

Em suma, considerando os dois modelos, o teste de Causalidade de Granger apontou que as variáveis tcpibbrasil, tcpibadvecon, juros, cambio, djia_per e todas em conjunto ajudam a explicar o comportamento dos fluxos de IED no Brasil.

\subsection{Análise de Decomposição da Variância (ADV)}

Segundo Bueno (2011), a análise de decomposição da variância (ADV) é uma forma alternativa de análise que indica qual a porcentagem da variância do erro previsão que decorre de cada variável endógena do modelo ao longo do horizonte de previsão. Isso posto, o uso da ADV nesse trabalho tem por objetivo avaliar a contribuição relativa das diversas variáveis para o comportamento dos fluxos de IED no Brasil, no período 1980-2010. A tabela 6 sintetiza os resultados da ADV para o modelo I, e a tabela 7 mostra os resultados para o modelo II.

Tabela 6 - ADV para IED Líquido - Modelo I (1980-2010)

\begin{tabular}{ccccccc}
\hline $\begin{array}{c}\text { Modelo I } \\
\text { Período }\end{array}$ & died_liq & tcpibbrasil & tcpibadvecon & djuros & dtcambio & dinf_CPI \\
\hline 1 & 100 & 0 & 0 & 0 & 0 & 0 \\
4 & 55.6154 & 16.3481 & 16.7935 & 5.5056 & 5.6895 & 0.0479 \\
8 & 45.3755 & 13.4863 & 30.1146 & 4.8081 & 6.0897 & 0.1259 \\
\hline
\end{tabular}

Os resultados da ADV para o Brasil, utilizando o modelo I, revelam uma enorme predominância da taxa de crescimento real do PIB nas economias avançadas (tcpibadvecon) na explicação da variância dos fluxos de IED, com uma participação de 30,1\% ao final do oitavo período, seguida pela taxa de crescimento real do produto interno (tcpibbrasil) com uma importância $16,3 \%$ ao final do quarto período, que se reduz para $13,5 \%$ ao final do oitavo período. Ressalta-se que os próprios fluxos de IED representam cerca de $50 \%$ das variações dos fluxos desse investimento ao longo do tempo. A taxa de juros e a taxa de câmbio, por sua vez, representam cerca de 5 e $6 \%$ das variações nos fluxos de IED, enquanto a inflação segue de longe, com $0,12 \%$ ao final do oitavo período.

Tabela 7 - ADV para IED Líquido - Modelo II (1980-2010)

\begin{tabular}{ccccc}
\hline $\begin{array}{c}\text { Modelo II } \\
\text { Período }\end{array}$ & died_liq & djia_per & ibov_per & dEFI \\
\hline 1 & 100 & 0 & 0 & 0 \\
4 & 70.5094 & 14.7879 & 8.6026 & 6.1002 \\
8 & 63.3057 & 19.8805 & 11.285 & 5.5288 \\
\hline
\end{tabular}

Ordem de Cholesky: died_liq djia_per ibov_per dEFI

Fonte: Stata12. Elaboração própria. 
Observando o segundo modelo, constata-se que os próprios fluxos de IED tem uma participação ainda maior nas variações dos fluxos desse investimento ao longo do tempo, com $70,5 \%$ ao final do quarto período, e $63,3 \%$ no oitavo. Os demais componentes apresentaram participações relativamente distribuídas, com ligeiro destaque para o índice Dow Jones, cujas variações explicam cerca de $20 \%$ das variações dos fluxos de IED ao final do período 8 , indicando que o dinamismo dos mercados de capitais nos EUA é um forte determinante dos fluxos de saída desses investimentos, sobretudo nos últimos anos. Na sequência, as variações no IBOV respondem por cerca de $11 \%$ das flutuações nos influxos de IED no longo prazo, e o índice de Liberdade Econômica (EFI), entre 5 e $6 \%$.

\subsection{Análise das funções Impulso-resposta (IRF)}

O efeito dos choques das variáveis do modelo I sobre o fluxo de IDE líquido para a economia brasileira durante o período de 1980 a 2010 é apresentado na figura 3.

No primeiro quadro é apresentado o resultado de um choque na própria variável, no segundo quadro apresenta-se a resposta de um choque da taxa de crescimento do Brasil, o terceiro quadro mostra a resposta de um choque da variável taxa de crescimento das economias avançadas, o quarto quadro apresenta o resultado de um choque da variável taxa de juros, o quinto quadro mostra o resultado de um choque da variável taxa de câmbio e no sexto quadro visualiza-se um choque da variável inflação. As funções resposta ao impulso foram estimadas para um horizonte de 8 anos.

A figura 3, mostra que um choque nas variáveis: fluxo de IED líquido e taxa de crescimento das economias avançadas apresentaram um efeito inicial positivo sobre o fluxo de IED líquido, sendo que no caso do fluxo de IED líquido o choque não produz efeitos duradouros. Já para um choque nas variável taxa de crescimento do Brasil, inicialmente há uma resposta negativa, mas esta reverte-se já no período subsequente. No caso da taxa de juros, taxa de câmbio e na inflação, elevações destas tendem a surtir um efeito negativo sobre os fluxos de IED líquido. 
Figura 3 - Funções Impulso-Resposta - Modelo I

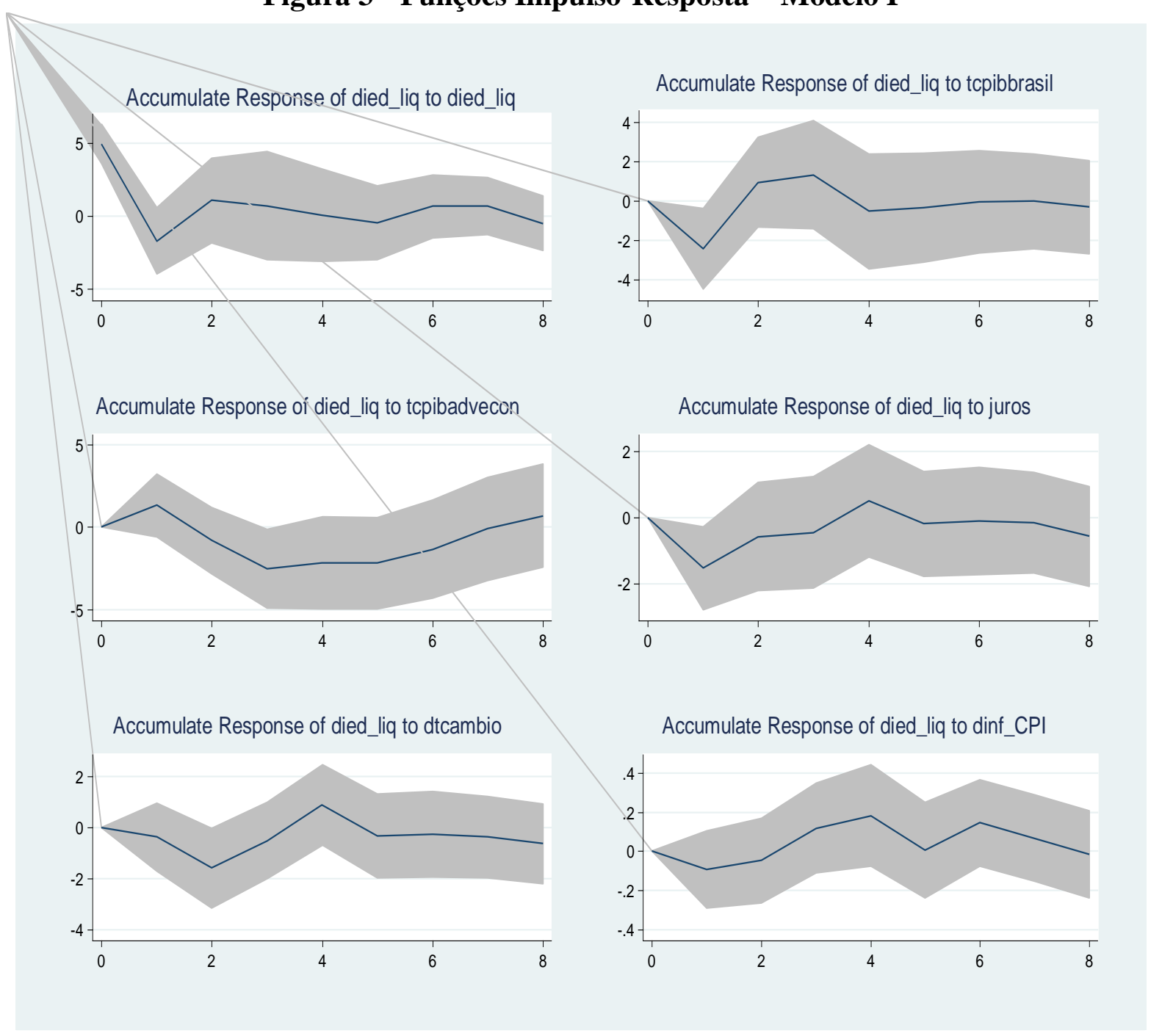

Fonte: Stata12. Elaboração própria.

O efeito dos choques das variáveis do modelo II sobre o fluxo de IDE líquido para a economia brasileira durante o período de 1980 a 2010 é apresentado na figura 4. No primeiro quadro é apresentado o resultado de um choque na própria variável, no segundo quadro apresenta-se a resposta de um choque na variável variação do Índice BOVSPA, o terceiro quadro mostra a resposta de um choque da variável variação do Índice DOW JONES, o quarto quadro apresenta o resultado de um choque da variável liberdade econômica. As funções resposta ao impulso foram estimadas para um horizonte de 8 anos. 


\section{Figura 4 - Funções Impulso-Resposta - Modelo II}

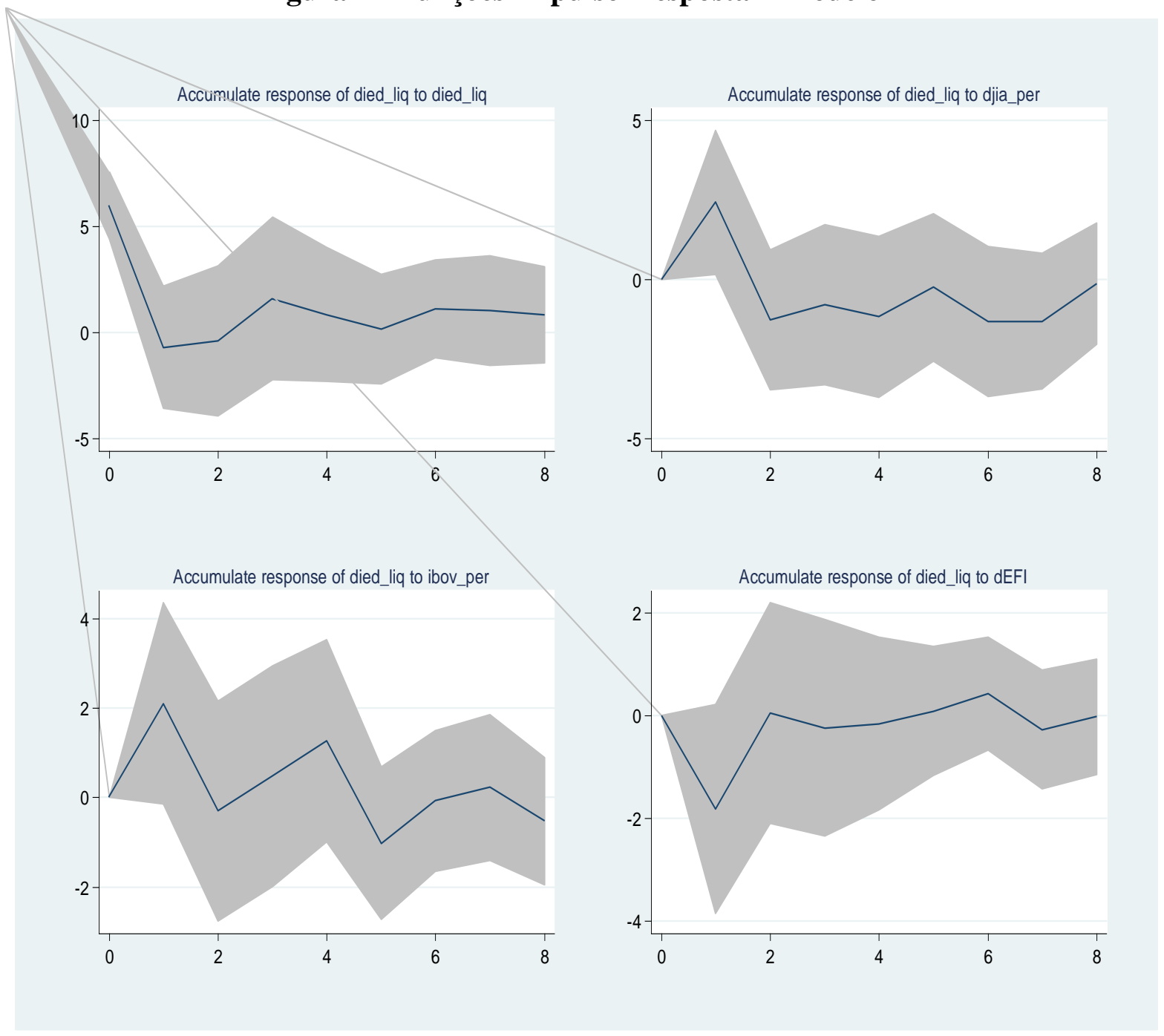

Fonte: Stata12. Elaboração própria.

Como mostra a figura 4, um choque nas variáveis: fluxo de IED líquido, variação do Índice BOVESPA e variação do Índice DOW JONES apresentaram um efeito inicial positivo sobre o fluxo de IED líquido, que é mitigado ao longo do tempo. Já para um choque na variável liberdade econômica, observou-se um efeito inicial negativo sobre os fluxos de IED líquido, que reverte-se rapidamente nos períodos seguintes.

\section{Conclusão}

Este trabalho teve como objetivo analisar os determinantes do fluxo de investimento estrangeiro direto para o Brasil desde 1980, no contexto tradicional dos fatores push-pull, uma vez que o influxo de grande quantidade dessa modalidade de investimento tende a criar efeitos significativos sobre o desempenho econômico nos países receptores. Para tal propósito, a análise utiliza dois modelos recursivos de Vetores Autorregressivos (VAR), abarcando o período 1980-2010.

Fatores domésticos (pull factors) empregados no trabalho como: taxa de crescimento real do PIB nacional, taxa de câmbio nominal, índice de preços do mercado de ações 
(Ibovespa), índice de Liberdade Econômica e, em menor grau, a taxa de juros Selic, mostraram-se importantes quando se pretende analisar os determinantes da entrada de IED no Brasil. Do lado externo (push factors), a taxa de crescimento real do produto das economias avançadas e o índice de ações das 30 maiores empresas norte-americanas, o DJIA, também revelaram-se importantes para explicar as variações nos fluxos de IED.

Em relação à resposta aos choques, destaca-se que, sob a ótica mais curto-prazista, a taxa de crescimento do PIB doméstico é mais relevante na explicação desses fluxos de capitais estrangeiros, enquanto num horizonte temporal mais estendido, o crescimento do PIB nas economias avançadas é predominante. Da mesma forma, choques nas taxa de câmbio e de juros, e nos índices BOVESPA, DJIA e de Liberdade Econômica provocam uma resposta mais rápida dos fluxos de IED, enquanto a inflação, representada pelo IPC, tende a levar um tempo maior.

\section{REFERÊNCIAS}

BUENO, R. Econometria de séries temporais. 2a Ed. São Paulo: Cengage Learning, 2011.

BUCKEY, P. J., CASSON, M. The future of the multinational enterprise. London: MacMillan, 1976. 116p.

BUCKEY, P. J., CASSON, M. The optimal timing of a foreign direct investment. Economic Journal, v.91, n.361, p.75-87, Mar.1981.

CAMPOS, N. F., KINOSHITA, Y. Why does FDI go where it goes? New evidence from the transition economies. Washington: International Monetary Fund Institute, 2003. 31p. (Working Paper; 03/228).

CANTWELL, J., NARULA, R. The eclectic paradigm in the global economy. International Journal of the Economics of Business, v.8, n.2, p.155-172, 2001.

CAVES, R. E. International corporations: the industrial economics of foreign investment. Economica, v.38, n.149, p.1-27, Fev. 1971.

COSTA, C. G. Factores de atracção do investimento directo estrangeiro para a economia brasileira nos anos 90 e as repercussões sobre a especialização internacional no Brasil. Informação Internacional: análise econômica e política, p.53-77, Ministério do Ambiente e do Ordenamento Territorial de Portugal, 2002.

DUNNING, J. H. The eclectic (OLI) paradigm of international production: past, present and future. International Journal of the Economics of Business, v.8, n.2, p.173-190, 2001.

DUNNING, J. H. Multinational enterprises and the global economy. Reading, Mass.: Addison-Wesley, 1993. 687p.

DUNNING, J. H. (1988), "Trade, location of economic activity and the multinational enterprise: a search for an eclectic approach". In Dunning, J. H. (comp.), Explaining international production, London, p. 13-40, Unwin Hyman. 
DUNNING, J. H., KIM, C., LIN, J. Incorporating trade into the investment development path: a case study of Korea and Taiwan. Oxford Development Studies, v.29, n.2, p.145-154, 2001.

ELLIOTT, G; ROTHENBERG, T.J; STOCK, J.H. (1996). Efficient tests for an autoregressive unit root. Econometrica, 64, pp.813-836.

ENDERS, W. Applied econometric time series. New York: John Wiley \& Sons, Inc, 1995.

FMI - Fundo Monetário Internacional. Disponível em: <http://www.imf.org>. Acesso em: nov, 2012.

FRASER INSTITUTE. Web site of the Economic Freedom Network. Disponível em: http://www.freetheworld.com/. Acesso: Nov/2012.

GARIBALDI, P. et alii. What moves capital to transition economies? IMF Staff Papers, v.48, Special Issues, p.109-145, 2001.

GONÇALVES, R. Globalização e desnacionalização. São Paulo: Paz e Terra, 1999.

GRAHAM, E. M. Market structure and the multinational enterprise: a game-theoretic approach. Journal of International Business Studies, v.29, n.1, p.67-83, 1998.

GRAHAM, E. M. Transatlantic investment by multinational firms: a rivalistic phenomenon? Journal of Post Keynesian Economics, v.1, n.1, p.82-99, 1978.

GREENE, W. H. Econometric Analysis. 7ª ed. New Jersey: Pearson Prentice Hall, 2011.

HOLLAND, D. et alii. The determinants and impact of FDI in central and eastern Europe: a comparison of survey and econometric evidence. New York: Transnational Corporations, v. 9, n. 3, Dec, 2000.

HYMER, S. H. Empresas multinacionais: a internacionalização do capital. Rio de Janeiro: Graal, 1978. Capítulo 1. O impacto das empresas internacionais. p.11-35.

HYMER, S. H. The international operations of national firms: a study of direct foreign investment. (Ph.D. Dissertation). p. 253, 1976.

HYMER, S. H. The international operations of national firms. Cambridge, The MIT Press, 1960.

IPEADATA. Base de dados econômicos e financeiros mantida pelo Instituto de Pesquisa Econômica Aplicada. Disponível em: http://www.ipeadata.gov.br/. Acesso: Nov/2012.

JABBOUR, E; ACIOLY, L; MIGUEL, S. I Seminário Bric: Oportunidades e Desafios: Breves notas comparativas em economia e história (2006). Disponível em: http://www2.camara.gov.br/eve/realizados/bric/programacao.html. Acesso: Jan/2013.

KINDLEBERGER, C. P. American business abroad: six lectures on direct investment. New Heaven: Yale University, 1969. 225p.

LACERDA, A. C. Globalização e Investimento Estrangeiro no Brasil. São Paulo: Editora Saraiva, 2004. 
LACERDA, A. C. (Org.). Desnacionalização: mitos, riscos e desafios. São Paulo: Editora Contexto, 2000.

LACERDA, A. C. O Impacto da globalização na economia brasileira. São Paulo: Editora Contexto, 1998, 1ª edição.

LANE, P. R., MILESI-FERRETTI, G. M. External capital structure: theory and evidence. London: Centre for Economic and Policy Research, Discussion Paper 2583, 2000.

LIMA JÚNIOR, A. J. M. Determinantes do Investimento Direto Estrangeiro no Brasil. (Tese de Doutorado). UFMG/Cedeplar. Belo Horizonte, 2005.

MATTOS, L. B. et al. Determinantes dos investimentos diretos estrangeiros no Brasil, 19802004. Revista de Economia Contemporânea, p. 39-60, jan./abr, Rio de Janeiro, 2007.

NONNENBERG, M. J. B.; MENDONÇA, M. J. C. Determinantes dos investimentos diretos externos em países em desenvolvimento. Estudos Econômicos, v. 35, n. 4, p. 631-655, out/dec, São Paulo, 2005.

NUNNENKAMP, P., SPATZ, J. Determinants of FDI in developing countries: has globalization changed the rules of the game? Transnational Corporations, v.11, n.2, p.1-34, Aug.2002.

PHILLIPS, P.; PERRON, P. Testing for a unit root in time series regression. Biometrika, 75, 335-46, 1998.

NELSON, C. R.; PLOSSER, C. I. Trends and random walks in macroeconomic time series. Journal of Monetary Economics, v. 10, p. 139-162, 1982.

SCHNEIDER, F., FREY, B. S. Economic and political determinants of foreign direct investment. World Development, v.13, n.2, p.161-175, 1985.

SIMS, C. Macroeconomics and reality. Econometrica, v.48, n.1, p.1-48, 1980.

VERNON, R. International investment and international trade in the product cycle. Quarterly Journal of Economics, v.80, n.2, p190-207, 1966. 